\title{
The role of ultrasonography in the evaluation of portal hemodynamics in healthy adults and pathologic conditions
}

1. Faculty of Medicine, University "Ovidius" of Constanta

2. Gastroenterology Department, Clinical Emergency County Hospital „Sf Apostol Andrei”, Constanta

\begin{abstract}
Introduction: Portal hypertension results from increased resistance to the portal blood flow. The ultrasound represents a noninvasive tool for assessing the structural and hemodynamic abnormalities in this condition.

Objectives: The assessment of the hemodynamic blood flow of the liver and spleen by using Doppler ultrasound in patients with portal hypertension compared with healthy subjects and to identify correlations with the severity of liver cirrhosis and esophageal varices.

Materials and Method: This study included 50 patients with liver cirrhosis and 20 healthy subjects as controls. Different correlations were carried out between hemodynamic variables obtained from the Doppler examination and the severity of cirrhosis as assessed by the Child score and the degree of esophageal varices at endoscopy.

Results: The portal vein diameter was larger in cirrhotic patients compared with the control group. The mean portal vein velocity and the blood flow were significantly lower in advanced forms of liver cirrhosis. The portal vein congestion index, the pulsatility and resistivity indexes of the hepatic artery were significantly increased in patients with CHILD B and C cirrhosis compared with the control group and patients in CHILD class A. There were no statistically significant differences between the
\end{abstract}

Eugen Dumitru

145 Tomis Boulevard, Clinical Emergency County Hospital Constanta, Internal Medicine I, Department of Gastroenterology

Constanta, Romania

email: eugen.dumitru@yahoo.com

phone: +40744761454 pulsatility and resistivity indexes of the splenic artery in patients with cirrhosis. The liver vascular index was significantly lower in patients with advanced cirrhosis. Conclusions: The ultrasound is a valuable noninvasive tool for cirrhotic patients with portal hypertension. However, it is not sufficiently accurate in differentiating between different classes of cirrhosis or degrees of esophageal varices.

Keywords: portal hypertension, portal vein (PV), hepatic artery (HA), splenic artery (SA), congestion index $(\mathrm{CI})$, pulsatility index (PI), resistivity index (RI), PVV (portal vein velocity), liver vascular index (LVI)

\section{Introduction}

Portal hypertension results from increased resistance to the portal blood flow. On the other hand, the portal blood flow increases due to splanchnic vasodilation. Both of these mechanisms contribute to the progression of portal hypertension [1,2].

The severity of portal hypertension is determined by measuring the hepatic venous pressure gradient (HVPG) [3]. This is the gold standard measurement; however, it is invasive and available only in a few centers. Therefore, there is a need to develop noninvasive methods of evaluating portal hypertension in cirrhotic patients. The ultrasound can yield important data about morphologic changes and liver hemodynamics [4]. 
Table I Patient characteristics of the study

The aim of this study is to assess the hemodynamic arterial and venous flow of the liver and spleen by using Doppler ultrasound in patients with liver cirrhosis and portal hypertension compared with healthy subjects and to identify specific correlations with the severity of liver cirrhosis and degree of esophageal varices.

\section{Material and Method}

We enrolled 50 patients with liver cirrhosis (31 men, $62 \%$; and 19 women, $38 \%$ ) with a mean age of $49.1 \pm 7.8$ years and 20 healthy subjects $(12 \mathrm{men}$, $60 \%$; and 8 women, $40 \%$ ) with a mean age $51.7 \pm 9.3$ years as the control group. The study was carried out from September 1, 2014 to June 30, 2015 (10 months). The patients were admitted to the Gastroenterology Department, Clinic of Internal Medicine I, Constanta.

The following exclusion criteria were applied: history or presence of hepatic encephalopathy; history of upper gastrointestinal bleeding, hepatocellular carcinoma, cardiopulmonary disease, hypertension, renal artery stenosis, diabetes mellitus, or chronic renal failure; prior acute therapies for portal hypertension; use of vasoactive drugs, diuretics, or anti-inflammatory medication; ongoing antiviral therapy; portal vein thrombosis; and age under 18 or over 70 years.

Laboratory tests were done, and the patients were grouped according to the Child classification: Group I (Child A), 14 patients; Group II (Child B), 21 patients; and Group III (Child C), 15 patients. Table I shows the characteristics of the study subjects.

Upper gastrointestinal endoscopy was carried out with a Pentax EPK-100p digital video endoscopy. We looked for the presence of esophageal varices and their degree according to the Japanese classification (Japanese Research Society for Portal Hypertension)

\begin{tabular}{|l|c|c|}
\hline & Patients & Control group \\
\hline Number & 50 & 20 \\
\hline $\mathrm{M}: \mathrm{F}$ & $1.63: 1$ & $1.50: 1$ \\
\hline Age (years) & $49.1 \pm 7.8$ & $51.7 \pm 9.3$ \\
\hline Child class A / B / C & $14 / 21 / 15$ & - \\
\hline The etiology of & $\begin{array}{l}\text { - Alcohol: } 14 \\
\text { cirrhosis }\end{array}$ & $\begin{array}{l}\text { - HCV: } 21 \\
\text { - VHB: } 9 \\
\text { - Mixed: } 6\end{array}$ \\
\hline
\end{tabular}

Doppler ultrasonography was done with a Hitachi Aloka ProSound Alpha 7 device with a UST-9147 multi-frequency convex transducer. The examination was carried out on patients under fasting and in a supine position for 15 minutes. The Doppler sample was positioned in the middle of the lumen.

The following variables were calculated: portal vein diameter, sectional area and velocity (minimum, maximum, and average), portal vein flow, resistivity and pulsatility indexes of the hepatic and splenic arteries, congestion index of the portal vein, and liver vascular index (LVI).

a) Portal vein

1. Portal vein diameter (PVD; $\mathrm{mm}$ )

The portal vein was measured at the hepatic hilum, proximal to the bifurcation.

2. Cross-sectional area of the portal vein $(\mathrm{cm} 2)$

Cross sectional area $=\left(\pi \times\right.$ diameter $\left.^{\wedge} 2\right) / 4$

3. Mean portal vein velocity $(\mathrm{cm} / \mathrm{sec})(\mathrm{PVV})$

Hemodynamic measurements were done during a brief apnea after a small breath. The insonation angle between the vessel and Doppler beam was less than 600 .

4. Portal venous flow (PVF)

Flow $=\left[\left(\pi \times\right.\right.$ diameter $\left.\left.^{\wedge} 2\right) / 4\right] \times$ mean velocity

b) Hepatic artery pulsatility index (HAPI) and splenic artery pulsatility index (SAPI)

$\mathrm{PI}=($ Peak sistolic velocity - end diastolic velocity)/(Mean velocity)

c) Hepatic artery resistive index (HARI) and splenic artery resistive index (SARI)

$\mathrm{RI}=($ Peak sistolic velocity - end diastolic velocity)/(Peak sistolic velocity) 
d) Congestion index of the portal vein (CI)

$\mathrm{Cl}=$ (cross-sectional area of the portal vein)/ (mean portal vein velocity)

e) Liver vascular index (LVI)

$\mathrm{LVI}=($ Portal vein velocity $) / H A P I$

We carried out different correlations between the hemodynamic variables obtained from the Doppler examination and the severity of cirrhosis as assessed by the Child score and the degree of esophageal varices.

The results for different groups of patients were compared by applying the nonparametric unpaired Mann-Whitney U test (for two groups) and the Kruskal-Wallis test (for more than two groups). The results were considered significant at $\mathrm{p}<0.05$. Statistical analysis was done with the Microsoft Excel 2007 software.

\section{Results}

Table II shows a summary of the abovementioned anatomic and hemodynamic (functional) variables. The portal vein diameter was larger in cirrhotic patients compared with the control group. However, this variable was not correlated with the severity of liver cirrhosis according to the CHILD class.
The mean portal vein velocity was significantly lower in patients with cirrhosis compared with the control group, and there was a decrease in velocity with increasing severity of liver cirrhosis. The lowest velocities were in CHILD classes B and C.

The blood flow through the portal vein was significantly decreased only in advanced forms of liver cirrhosis (Child C), and there were no significant differences between the control group and patients with compensated cirrhosis (Child A). CHILD B patients with cirrhosis showed a decrease in portal blood flow, but the value was not significant.

The portal vein congestion index (CI) was increased in patients with liver cirrhosis compared with the control group, and patients with decompensated cirrhosis (Child C) showed the highest increase in this index.

The pulsatility and resistivity indexes of the hepatic artery were significantly increased in patients with CHILD B and C cirrhosis compared with the control group and patients in CHILD class A.

The analysis of the splenic artery Doppler indexes showed no statistically significant differences between the pulsatility index (PI) and resistivity index (RI) of the splenic artery in patients with hepatic cirrhosis compared with the control group, as well as between these indicators and the CHILD classes of liver cirrhosis.

The liver vascular index (LVI) was significantly lower in patients with advanced cirrhosis (CHILD C) compared to compensated cirrhosis patients (CHILD A) and controls. Although LVI was lower also in

Table II. 2D ultrasound and Doppler values in cirrhotic patients compared with controls

\begin{tabular}{|l|c|c|c|c|}
\hline Parameter & Control group & CHILD A & CHILD B & CHILD C \\
\hline PV Diameter (mm) & $11.2 \pm 1.8^{*}$ & $13.9 \pm 1.4$ & $14.6 \pm 2.1$ & $14.2 \pm 1.9$ \\
\hline PV velocity $(\mathrm{cm} / \mathrm{sec})$ & $19.1 \pm 3.2$ & $16.5 \pm 2.1$ & $12.1 \pm 1.9^{*}$ & $10.2 \pm 2.8^{*}$ \\
\hline PV flow rate (mL/ sec) & $1253.7 \pm 65.2$ & $1279.3 \pm 53.7$ & $1126.1 \pm 98.5$ & $857.2 \pm 86.8^{*}$ \\
\hline Congestion index of PV (cm x sec) & $0.086 \pm 0.011^{*}$ & $0.113 \pm 0.023$ & $0.121 \pm 0.025$ & $0.175 \pm 0.028^{*}$ \\
\hline Pulsatility index of HA & $1.03 \pm 0.07$ & $1.09 \pm 0.08$ & $1.38 \pm 0.12^{*}$ & $1.72 \pm 0.18^{*}$ \\
\hline Resistivity index of HA & $0.54 \pm 0.06$ & $0.57 \pm 0.07$ & $0.71 \pm 0.07^{*}$ & $0.78 \pm 0.06^{*}$ \\
\hline Pulsatility index of SA & $0.92 \pm 0.08$ & $0.99 \pm 0.11$ & $0.94 \pm 0.09$ & $1.03 \pm 0.15$ \\
\hline Resistivity index of SA & $0.475 \pm 0.065$ & $0.469 \pm 0.078$ & $0.533 \pm 0.069$ & $0.561 \pm 0.072$ \\
\hline Liver vascular index (LVI) & $17.52 \pm 2.76$ & $15.08 \pm 2.63$ & $10.14 \pm 3.98$ & $8.36 \pm 3.13^{*}$ \\
\hline
\end{tabular}

Results were compared for each group with both other groups, independently. $* \mathrm{P}<0.05$ 
Table III 2D and Doppler ultrasound values in patients with liver cirrhosis depending on the size of esophageal varices

\begin{tabular}{|l|c|c|c|}
\hline Parameter & EV grade I & EV grade II & EV grade III \\
\hline PV Diameter (mm) & $14.1 \pm 2.5$ & $14.2 \pm 2.3$ & $14.6 \pm 2.4$ \\
\hline PV velocity (cm / sec) & $12.3 \pm 2.3$ & $10.6 \pm 1.9$ & $10.4 \pm 2.5$ \\
\hline PV flow rate (mL/ sec) & $1119.4 \pm 92.8$ & $871.2 \pm 88.2$ & $843.4 \pm 91.3^{*}$ \\
\hline Congestion index of PV (cm x sec) & $0.142 \pm 0.031$ & $0.123 \pm 0.027$ & $0.178 \pm 0.034^{*}$ \\
\hline Pulsatility index of HA & $1.56 \pm 0.14$ & $1.49 \pm 0.11$ & $1.28 \pm 0.15$ \\
\hline Resistivity index of HA & $0.69 \pm 0.06$ & $0.72 \pm 0.05$ & $0.76 \pm 0.08$ \\
\hline Pulsatility index of SA & $1.02 \pm 0.09$ & $0.91 \pm 0.08$ & $1.07 \pm 0.09$ \\
\hline Resistivity index of SA & $0.495 \pm 0.058$ & $0.519 \pm 0.061$ & $0.526 \pm 0.055$ \\
\hline Liver vascular index (LVI) & $11.71 \pm 2.9$ & $10.27 \pm 3.67$ & $8.21 \pm 3.20 *$ \\
\hline
\end{tabular}

Results were compared for each group with both other groups, independently. ${ }^{*} \mathrm{P}<0.05$

CHILD B class, the value did not have statistical significance.

Table III shows the values obtained in the $2 \mathrm{D}$ and Doppler ultrasound in relation to the size of esophageal varices. In general, these variables correlated with the severity of esophageal varices (EV), except for PV flow and LVI, which were significantly decreased in patients with EV grade III.

\section{Discussion}

The identification of prognostic factors associated with the risk of decompensation of liver cirrhosis and of gastrointestinal bleeding in esophageal varices is a long-standing objective in medicine. The ultrasound is a relatively inexpensive and noninvasive method that has undergone considerable development in medical practice and has recently benefited from significant technical improvements. Therefore, it is justified to look for a correlation between the ultrasound parameters and the evolution and prognosis of cirrhotic patients.

The results of this study showed that the portal vein diameter was significantly higher in cirrhotic patients compared with the control group, similarly to other works [6]. However, the portal vein diameter was not useful in differentiating between grades of severity of cirrhosis, nor was it correlated with the size of esophageal varices. A study in 2012 [7] also showed a weak and useless correlation in practice between the portal vein diameter and the liver cirrhosis CHILD or MELD score although some studies have reported otherwise [8]. Our results indicate that this variable cannot be used in clinical practice to assess bleeding risk or to make a prognosis for patients with cirrhosis, possibly because the diameter of the portal vein depends on the number and size of the collateral venous portal system, which has large individual variability.

The velocity through the portal vein was significantly decreased in patients with decompensated cirrhosis (Child B and C) compared with the control group and patients in Child class A, as similarly observed in other studies $[6,8,9]$. Gaiani [10], among other authors, reported similar results: the velocity of the portal vein was significantly lower in cirrhotic patients compared with controls and was significantly lower in patients with Child B and C cirrhosis than those with Child A (Table IV).

\begin{tabular}{|l|c|c|}
\hline \multirow{2}{*}{} & \multicolumn{2}{|c|}{ Mean portal velocity $(\mathrm{cm} / \mathrm{sec})$} \\
\cline { 2 - 3 } & Control & Cirrhosis \\
\hline Gaiani et al [10] & $16 \pm 4.1$ & $12.4 \pm 2.3$ \\
\hline Moriyasu et al [11] & $15.3 \pm 4$ & $9.7 \pm 2.6$ \\
\hline Zoli et al [12] & $16 \pm 0.5$ & $10.2 \pm 3.5$ \\
\hline Ohnishi et al [13] & $16.5 \pm 4.9$ & $12 \pm 3$ \\
\hline
\end{tabular}

The velocity through the portal vein (PVV) is not a constant variable correlated with the severity 
of cirrhosis, although some studies have reported otherwise [7,14]. The inconsistent results obtained are due to the existence of a certain intra- and interobserver variability and venous collaterals [15].

The blood flow through the the portal vein (PVF) depends on the PV diameter (which increases with the severity of cirrhosis) and the velocity through the PV (which decreases with the severity of cirrhosis). Therefore, the flow through the PV does not show significant changes over the long-term evolution of cirrhotic patients. However, in advanced stages and patients with grade III varices, the PV flow was significantly decreased, thus confirming the usefulness of this variable in identifying patients with poor prognosis, increased risk of decompensation, and digestive hemorrhage. A similarly useful variable is the congestion index of the portal vein, which was significantly increased in patients with CHILD C cirrhosis and those with grade III varices both in the present study and in previous works $[7,11]$.

We observed a significant increase in the hepatic artery pulsatility and resistivity indexes (HAPI and HARI) in patients with liver cirrhosis compared with the control group, similarly to other studies [6]. Moreover, these indexes were correlated with the size of esophageal varices but not with the severity of cirrhosis (Child classes) [16].

Sacerdoti et al. [16] found that the hepatic artery PI and RI measured intrahepatically were significantly higher in cirrhotic patients compared with a control group. The hepatic arterial impedance indexes were higher in some cirrhotic patients with portal vein thrombosis than in those without thrombosis.

Bolognesi et al. (1996) evaluated the splenic Doppler impedance indexes in patients with portal hypertension and a control group. They found that the pulsatility index was higher in cirrhotic patients with portal vein thrombosis than in those without thrombosis [17].

The liver vascular index (LVI) was significantly lower both in patients with decompensated cirrhosis (Child $\mathrm{C}$ ) and in those with esophageal varices at high risk of bleeding (Grade III), thus identifying this index as a possible marker of gravity that needs to be monitored regularly, as previously reported in the literature [6].

In our study, we did not find a good correlation between the size of esophageal varices and any of the static or hemodynamic ultrasound variables, except in the group of patients with grade III varices, who had significantly low blood flow through the portal vein and liver vascular index (LVI) and significantly increased portal vein congestion index. These results, which correspond to previous findings in the literature [18], were attributed to the existence of portosystemic anastomoses, which drain the portal circulation to other vascular territories not only through esophageal varices [19].

\section{Conclusions}

The standard 2D and Doppler ultrasound is an important, noninvasive screening tool for cirrhotic patients with portal hypertension. Significant differences are found between the static and impedance indexes of portal circulation in healthy subjects compared with those with portal hypertension. However, these indexes are insufficient to establish a survival prognosis for patients or determine the severity of esophageal varices. Therefore, there is a need to combine clinical scores with biological and imaging tools for a better assessment of these patients.

\section{References}

1. Iwakiri, Y. (2014). Pathophysiology Of Portal Hypertension. Clin Liver Dis. 18(2), 281-291. Doi: 10.1016/J.Cld.2013.12.001

2. Martell, M., Coll M., Ezkurdia, N., Raurell, I. \& Genescà, J. (2010). Physiopathology Of Splanchnic Vasodilation In Portal Hypertension. World J Hepatol. 2(6), 208-220. Doi: 10.4254/ Wjh.V2.I6.208

3. Wiest, R. \& Groszmann, R.J. (2002). The 
Paradox Of Nitric Oxide In Cirrhosis And Portal Hypertension: Too Much, Not Enough. Hepatology. 35, 478-91. Doi: 10.1053/ Jhep.2002.31432

4. Tchelepi, H., Ralls, P.W., Radin, R. \& Grant, E. (2002). Sonography Of Diffuse Liver Disease. Journal Of Ultrasound In Medicine. 21(9), 10231032.

5. Tajiri, T., Yoshida, H., Obara, K. \& Onji, M. (2010). General Rules For Recording Endoscopic Findings Of Esophagogastric Varices. Digestive Endoscopy. 22(1), 1-9.

6. Haktanir, A., Cihan, B.S., Çelenk, Ç. \& Cihan, S. (2005). Value Of Doppler Sonography In Assessing The Progression Of Chronic Viral Hepatitis And In The Diagnosis And Grading Of Cirrhosis. J Ultrasound Med. 24, 311-321

7. Shateri, K., Mohammadi, A., Moloudi, F., Nosair, E. \& Ghasemi-Rad, M. (2012). Correlation Between Sonographic Portal Vein Diameter And Flow Velocity With The Clinical Scoring Systems Meld And Ctp In Cirrhotic Patients: Is There A Relationship?. Gastroenterology Research. 5(3), 112-119

8. Yan, G., Duan, Y., Ruan, L., Chao, T. \& Yang, Y. (2004). A Study On The Relationship Between Ultrasonographic Score And Clinical Score (Meld, Cpt) In Cirrhosis. HepatoGastroenterology. 52(65), 1329-1333.

9. Chawla, Y., Santa Radha, N., Jang, K. D. \& Dilawari, B. (1998). Portal Hemodynamics By Duplex Doppler Sonography In Different Grades Of Cirrhosis. Digestive Diseases And Sciences, 43(2), 354-357. Doi: 10.1023/A:1018814624307.

10. Gaiani, S., Bolondi, L., Bassi, S. L., Santi, V., Zironi, G. \& Barbara, L. (1989). Effect On Meal On Portal Hemodynamics In Healthy Humans And In Patients With Chronic Liver Disease. Hepatology. 9(6), 815-819. Doi: 10.1002/ Hep.1840090604

11. Moriyasu, F., Nishida, O., Ban, N., Nakamura, T., Sakai, M., Miyake, T. \& Uchino, H. (1986). “ Congestion Index" Of The Portal Vein. American Journal Of Roentgenology, 146(4), 735-739. Doi: 10.2214/Ajr.146.4.735

12. Zoli, M., Dondi, C., Marchesini, G., Cordiani, M. R., Melli, A., \& Pisi, E. (1985). Splanchnic Vein
Measurements In Patients With Liver Cirrhosis: A Case-Control Study. Journal Of Ultrasound In Medicine, 4(12), 641-646.

13. Ohnishi, K., Saito, M., Sato, S., Nakayama, T., Takashi, M., Iida, S. \& Okuda, K. (1985). Direction Of Splenic Venous Flow Assessed By Pulsed Doppler Flowmetry In Patients With A Large Splenorenal Shunt: Relation To Spontaneous Hepatic Encephalopathy. Gastroenterology, 89(1), 180-185.

14. Schneider, A.R.J., Teuber, G., Kriener, S. \& Caspary, W. F. (2005). Noninvasive Assessment Of Liver Steatosis, Fibrosis And Inflammation In Chronic Hepatitis $\mathrm{C}$ Virus Infection. Liver International, 25(6), 1150-1155. Doi: 10.1111/J.1478-3231.2005.01164.X

15. Piscaglia, F., Donati, G., Cecilioni, L., Celli, N., Stagni, B., Pini, P. \& Bolondi, L. (2002). Influence Of The Spleen On Portal Haemodynamics: A Non-Invasive Study With Doppler Ultrasound In Chronic Liver Disease And Haematological Disorders. Scandinavian Journal Of Gastroenterology, 37(10), 12201227.

16. Sacerdoti, D., Merkel, C., Bolognesi, M.,Amodio, P., Angeli, P. \& Gatta, A. (1995). Hepatic Arterial Resistance In Cirrhosis With And Without Portal Vein Thrombosis: Relationships With Portal Hemodynamics. Gastroenterology, 108(4), 11521158. Doi: 10.1016/0016-5085(95)90214-7

17. Bolognesi, M., Sacerdoti, D., Merkel, C., Gerunda, G., Maffei-Faccioli, A., Angeli, P. \& Gatta, A. (1996). Splenic Doppler Impedance Indices: Influence Of Different Portal Hemodynamic Conditions. Hepatology, 23(5), 1035-1040. Doi: 10.1002/Hep.510230515

18. Zardi, E. M., Uwechie, V., Gentilucci, U. V., Dobrina, A., Petitti, T., Laghi, V. \& Afeltra, A. (2007). Portal Diameter In The Diagnosis Of Esophageal Varices In 266 Cirrhotic Patients: Which Role?. Ultrasound In Medicine \& Biology, 33(4), 506-511. Doi: 10.1016/J. Ultrasmedbio.2006.10.002

19. Taourel, P., Blanc, P., Dauzat, M., Chabre, M., Pradel, J., Gallix, B. \& Bruel, J. M. (1998). Doppler Study Of Mesenteric, Hepatic, And Portal Circulation In Alcoholic Cirrhosis: 
Relationship Between Quantitative Doppler Measurements And The Severity Of Portal Hypertension And Hepatic Failure. Hepatology, 28(4), 932-936. Doi: 10.1002/Hep.510280406 